\title{
DETERMINATION OF QUICK SWITCHING DOUBLE SAMPLING SYSTEM BY ATTRIBUTES UNDER FUZZY BINOMIAL DISTRIBUTION - SAMPLE SIZE TIGHTENING
}

\author{
K. Ramya ${ }^{1}$ and G. Uma ${ }^{2}$ \\ Department of Statistics, PSG College of Arts and Science, India
}

\begin{abstract}
Acceptance sampling is concerned with norms of deciding about the acceptance or rejection of the lots based on the quality of the product during inspection. Dodge and Romig popularized the acceptance sampling as a major field of SQC. Various Sampling plans, systems and schemes are developed as per the need of the industry. Quick Switching Systems is a system which requires fewer samples for processes running at low levels of defects. Quick Switching Systems are compared with single, double, multiple, chain and variable sampling plans as well as MIL STD 105 E switching systems and concluded its advantages. This article presents the Quick Switching Double Sampling System (QSDSS) when the fraction of non-conforming items is a fuzzy number and being modeled based on the fuzzy Binomial distribution. Operating Characteristic (OC) curves of the fuzzy system is like a band having high and low bounds whose width depends on the ambiguity proportion parameter in the lot when that sample size and acceptance numbers is fixed. Tables are constructed and numerical illustrations are given to describe the determination of QSDSS for sample size tightening with fuzzy Binomial distribution with its OC curve.
\end{abstract}

Keywords:

SQC, SSP, DSP, QSDSS, OC, Fuzzy Binomial Distribution

\section{INTRODUCTION}

An acceptance sampling plan is the overall scheme for accepting or rejecting a lot based on sample information. The acceptance plan identifies both the sample size and other criteria which are used to accept or reject the lot. Acceptance sampling plan is mostly used in industries to control the quality of products. Acceptance sampling is done in various phases like the incoming of the raw material, production process or even during the end of the production process. Sampling plans can be classified as single, double, multiple or sequential plans. Acceptance sampling plan has importance in the area of quality control and it can be applied when its requirements fulfilled. A combination of acceptance sampling plans with switching rules for changing from one plan to another are called acceptance sampling schemes. The simplest sampling scheme is Quick Switching System (QSS) introduced by Dodge and Romig [9] and further QSS was extensively studied by Romboski [22]. In this system, when a rejection occurs in normal inspection, an immediate switching to tightened inspection is made. QSS uses basic attribute sampling plans for lot inspections, called reference plan. QSDSS concentrate one's inspection effort where it will do the most good. Further, for processes running at low level of inspection but that react severely to the first hint of a problem.

E.G. Schiling [23] studied procedures and tables of Zero Acceptance Number Quick Switching System for Compliance Testing. Later Arumainayagam and Soundararajan [1] [2] analyzed QSS with double sampling plan as a reference plan (QSDSS) and constructed Quick Switching Double Sampling System for Sample Size Tightening and highlighted the advantages by comparing with Double Sampling Plan and Quick Switching System.

Palanivel [21] studied QSS with variable sampling plan as a reference plan. Soundararajan [18] have given procedure and tables for Quick Switching Variable Single sampling (QSVSS) systems indexed by AQL and AOQL. Uma and Arumainayagam [26] introduced Quick Switching Multiple Sampling System (QSMSS) for acceptance number tightening and constructed tables. Radhakrishnan [13] constructed and designed tables for QSDSS using Intervened Random Effect Poission Distribution (IRPD) through the parameters such as MAPD, MAAOQ and COAOQ. Suresh and Jayalakshmi [24] studied a procedure for selection of quick switching system with special type double sampling plans through Maximum Allowable Percent Defective and Maximum Allowable Average Outgoing Quality (MAPD and MAAOQ). Arumainayagam and Uma [7] have done a work on Construction and Selection of Quick Switching System Using Weighted Poisson distribution for sample size tightening.

This article shows how to evaluate and select Quick Switching System with reference to Double Sampling Plan (QSDSS) for sample size tightening using Fuzzy Binomial distribution. It introduces a new method of describing the production provided by QSDSS during periods of changing quality called transitive operating characteristic (OC) curves.

This paper is organized as follows: Section 2 discuss about acceptance sampling plans, section 3 gives an overview of operating characteristic curve, section 4 focuses on acceptance sampling and fuzzy set theory, section 5 focuses on quick switching double sampling systems. Section 6 explains the proposed Quick Switching Double Sampling System with Fuzzy Parameter using Binomial Distribution. Section 7 explains the OC Band with Fuzzy Parameter and section 8 gives a explanation with examples and section 9 describes about conclusion.

\section{ACCEPTANCE SAMPLING PLANS}

An acceptance sampling is a form of inspection applied to lots or batches of items before or after a process to judge conformance to predetermined standards. It is a decision making tool by which a conclusion is reached regarding the acceptability of lot. It is a quality control technique. Acceptance sampling is classified as attribute sampling plan and variable sampling plan. Attribute sampling plan is most commonly used when there are more than one type of quality characteristics considered for each sample. Variable sampling plan need measurement type data and decision must be based only on one such measurement characteristics. 
Sampling plans specify the lot size, sample size, number of samples and acceptance/rejection criteria. Sampling plans involve single sampling plan, double sampling plan, multiple sampling plan and sequential sampling plan.

\section{OPERATING CHARACTERISTIC CURVE}

A curve which serves to describe an acceptance plan in terms of probability of accepting lots of various quality levels with the plan. The operating characteristic (OC) curve describes how well an acceptance plan discriminates between good and bad lots. There are two risks involved with the operating characteristic curve, they are Producer's risk and Consumer's risk. Producer's risk is if the consumer rejects a batch of materials that are of good quality. Consumer's risk is accepting a batch of materials that are of poor quality but assuming the materials are of high quality.

\section{ACCEPTANCE SAMPLING AND FUZZY SET THEORY}

Fuzzy set theory represents an attractive tool to aid research in production in production management when the dynamics of the production environment limit the specification of model objectives, constraints and precise measurement of model parameters. Research on fuzzy quality management is broken down into three areas, acceptance sampling, statistical process control, and general quality management topics.

In different acceptance sampling plans the fraction of defective items, is considered as a crisp value, but in practice the fraction of defective items value must be known exactly. Many times these values are estimated or it is provided by experiment. The vagueness present in the value of $p$ from personal judgment, experiment or estimation may be treated formally with the help of fuzzy set theory. As known, fuzzy set theory is powerful mathematical tool for modeling uncertain resulting. In this basis defining the imprecise proportion parameter is as a fuzzy number. With this definition, the number of nonconforming items in the sample has a binomial distribution with fuzzy parameter. However if fuzzy number $p$ is small we can use the fuzzy poison distribution to approximate values of the fuzzy binomial.

Ohta and Ichihashi [20] present a fuzzy design methodology for single stage, two-point attribute sampling plans. An algorithm is presented an example sampling plans are generated when producer and consumer risk are defined by triangular fuzzy numbers. Chakraborty [5] examines the problem of determining the sample size and critical value of a single sample attribute sampling plan when imprecision exists in the declaration of producer and consumer risk. Earlier, a fuzzy goal programming model and solution procedure are described. Several numerical examples are provided and the sensitivity of the strength of the resulting sampling plans is evaluated. Earlier a paper details how possibility theory and triangular fuzzy numbers are used in the single sample plan design problem.

Kanagawa and Ohta [16] identify two limitations in the sample plan design procedure of Ohta and Ichihashi [16]. First, Ohta and Ichihashi's [16] design procedure does not explicitly minimize the sample size of the sampling plan. Second, the membership functions used, unrealistically model the consumer and producer risk. These deficiencies are corrected through the use of a nonlinear membership function and explicit incorporation of the sample size in fuzzy mathematical programming solution methodology. Chakraborty [6] [17] addresses the problem of designing single stage, Dodge-Roming [9] lot tolerance percent defective (LTPD) sampling plans when the lot tolerance percent defective, consumer's risk and incoming quality level are modeled using triangular fuzzy numbers. In the Dodge-Roming [9] scheme, the design of an optimal LTPD sample plan involves solution to a nonlinear integer programming problem. The objective is to minimize average total inspection subject to a constraint based on the lot tolerance percent defective and the level of consumer risk. When fuzzy parameters are introduced, the procedure becomes a possibility (fuzzy) programming problem.

Classical acceptance sampling plans have been studied by many researchers. Single Sampling by attributes with relaxed requirements were discussed by Ohta and Ichihashi [20] Kanagawa and Ohta [16], Tamaki et al. [25] Sampling plan by attributes for vague data were considered by Hryniewisz [14][15]. Jamkhaneh et al. [4] have studied acceptance Single Sampling Plan with fuzzy parameter using Poisson distribution. Jamkhaneh et al. [10] [12] have also studied the preparation important criteria of Rectifying Inspection for Single Sampling Plan with Fuzzy parameter. Gildeh et al. [3] have studied acceptance Double Sampling Plan with Fuzzy Paramter. Muthulakshmi and Malathi [19] analyzed a Special Double Sampling Plan with Fuzzy Parameter and constructed tables. Divya [8], analyzed Quality Interval Acceptance Single Sampling Plan with fuzzy parameter using Poisson distribution. Uma and Komaladevi [27] have undergone a work on Quick Switching System with Fuzzy Parameter using Poisson distribution. Turanoglu et al. [12] have designed Fuzzy acceptance sampling and operating characteristic curves. Uma and Devi [28] have studied Quick Switching System by attributes under Fuzzy Poisson Distribution and constructed tables for the ease use in the floor.

\section{QUICK SWITCHING DOUBLE SAMPLING SYSTEM}

Dodge [9] proposed a new sampling system consisting of pairs of normal and tightened plans with the switching rules constitute a sampling system. The application of system is as follows.

Step 1: Adopt a pair of sampling plans, a normal double sampling plan $N$ and tightened double sampling plan $T$ the plan to $T$ to be tighter $O C$ wiser than plan $N$.

Step 2: Use plan $N$ for the first lot.

Step 3: For each lot inspected: if the lot is accepted, use plan $N$ for the next lot; and if the lot is rejected, use plan $T$ for the next lot.

Step 4: Quick Switching Double Sampling System has the parameters $n\left(n_{1}=n_{2}=n\right)$, the sample size and $c_{1}, c_{2},\left(c_{1}<\right.$ $\left.c_{2}\right)$, the acceptance numbers $k,(k>1)$.

\subsection{CONDITIONS OF APPLICATION}

The conditions for application under which the Quick Switching System can be applied and the operation procedures are as follows: 
Step 1: The production is steady so that results on current and proceedings lots are broadly indicative of a continuing process and submitted lots are expected to be essentially of the same quality.

Step 2: Lots are submitted substantially in the order of production.

Step 3: Inspection is by attributes with quality defined as fraction nonconforming.

\subsection{OPERATING PROCEDURE OF QSDSS $\left(\mathbf{N}, \mathbf{K} ; \mathrm{C}_{1}, \mathrm{C}_{2}\right)$}

Step 1: From a lot, take a random sample of size $n$ and count the number of nonconforming units $X_{1}$.

(a) If $X_{1} \leq c_{1}$, accept the lot and repeat step 1 for the next lot.

(b) If $X_{1} \leq c_{2}$, reject the lot and go to step 2 .

(c) If $\mathrm{c}_{1}<X_{1} \leq c_{2}$, take a second random sample of size $n$ from the same lot and observe the number of nonconforming units $X_{2}$.

(d) If $X_{1}+X_{2} \leq c_{2}$, accept the lot and repeat step 1 for the next lot.

(e) If $X_{1}+X_{2}>c_{2}$, reject the lot and go to step 2 .

Step 2: From the next lot, take a random sample of size $n k$ and count the number of nonconforming units $X_{1}$.

(a) If $X_{1} \leq c_{1}$, accept the lot and go to step 1 for the next lot.

(b) If $X_{1} \leq c_{2}$, reject the lot and repeat step 2 for the next lot.

(c) If $\mathrm{c}_{1}<X_{1} \leq c_{2}$, take a random sample of size $n k$ from the same lot and observe the number of nonconforming units $X_{2}$.

(d) If $X_{1}+X_{2} \leq c_{2}$, accept the lot and repeat step 1 for the next lot.

(e) If $X_{1}+X_{2}>c_{2}$, reject the lot and go to step 2 for the next lot.

\subsection{MEASURES OF PERFORMANCE}

Using the concept of Markov chain, the OC function of QSS is derived by Romboski [22] (1969) as:

$$
P_{a}=\frac{P_{T}}{\left(1-P_{N}\right)+P_{T}}
$$

where, $P_{N}$ is the proportion of lots expected to be accepted when using normal double sampling plan $\left(n ; c_{1}, c_{2}\right)$ using Fuzzy Binomial and $P_{T}$ is the proportion of lots expected to be accepted when using normal double sampling plan $\left(n k ; c_{1}, c_{2}\right)$ using Fuzzy Binomial.

\section{QUICK SWITCHING DOUBLE SAMPLING SYSTEM WITH FUZZY PARAMETER USING BINOMIAL DISTRIBUTION}

If the size of the lot be large, the random variables $d_{1}$ and $d_{2}$ have Binomial distribution with parameters $\left(n_{1}, p\right)$ and $\left(n_{2}, p\right)$, in which $p$ indicates the fraction of the lot's nonconforming items. So, if we represent probability of acceptance on combined samples with $p_{\alpha}$, and also the probability of the lot's acceptance in first and second samples by $\tilde{P}_{a}^{I}, \tilde{P}_{a}^{I I}$, respectively, then

$$
P_{a}=P_{a}^{I}+P_{a}^{I I}
$$

where, $p_{a}^{I}$ indicates the probability of the event $d_{1} \leq c_{1}$. Thus,

$$
P_{a}^{I}=\sum_{d_{1}=0}^{c_{1}}\left(\begin{array}{l}
n_{1} \\
d_{1}
\end{array}\right) p^{d_{1}}(1-p)^{n_{1}-d_{1}}
$$

and $p_{a}^{I I}$ according to the independence of two random variables and their distributions will be calculated as follows:

$$
P_{a}^{I I}=P\left(d_{1}+d_{2} \leq c_{2}, c_{1}<d_{1}<c_{2}\right)
$$

Suppose that we want to inspect a lot with the size of ' $N$ ', in which the proportion of defective items or the probability of the defectiveness is not known precisely and it is an uncertain value. So we represent this parameter with a fuzzy number $\tilde{p}$ which is:

$$
\tilde{p}=\left(a_{1}, a_{2}, a_{3}\right), p \in \tilde{p}[1], q \in \tilde{q}[1], p+q=1
$$

A Quick Switching Double Sampling System with a fuzzy parameter is defined by the first sample size $n_{1}$, the acceptance number on the first stage $c_{1}$ the second sample size $n k$ and the acceptance number on the second stage $c_{2}$.

If the size of the lot was very great, the random variables $d_{1}$ and $d_{2}$ have a fuzzy Binomial probability distribution with parameters $n_{1} \tilde{p}$ and $n_{2} \tilde{p}$ in which $\tilde{p}$ indicates the fuzzy proportion of the defective items. According to this case, if we show the fuzzy probability of the acceptance of lot in the combined samples with $\tilde{P}_{a}$ and also the fuzzy probability of acceptance of the lot in the first and second samples, $\tilde{p}_{a}^{I}, \tilde{p}_{a}^{I I}$, respectively, then

$$
\tilde{p}_{a}=\tilde{p}_{a}^{I}+\tilde{p}_{a}^{I I}
$$

\section{OC BAND WITH FUZZY PARAMETER}

The OC curve represents the performance of the system by plotting the probability of acceptance of a lot versus its production quality, which is expressed by the proportion of nonconforming items in the lot. OC curve aids in selection of system that are effective in reducing risk and indicates discriminating power of the system.

The fuzzy probability of acceptance of a lot in terms of fuzzy fraction of defective items would be as a band with upper and lower bounds. The uncertainty degree of a proportion parameter is one of the factors that bandwidth depends on that. The less uncertainty value results in less bandwidth, and if proportion parameter gets a crisp value, lower and upper bounds will become equal, which that $\mathrm{OC}$ curve is in classic state. Knowing the uncertainty degree of proportion parameter and variation of its position on horizontal axis, we have different fuzzy number $(\tilde{p})$ and hence we will have different proportion $(p)$ which the OC bands are plotted in terms of it. 
Table.1. Probability of Acceptance for $\operatorname{QSDSS}_{F B}\left(n ; k ; c_{1} ; c_{2}\right)$

$n_{1}=n_{2}=n=35, k=1.25, c_{1}=1, c_{2}=2$

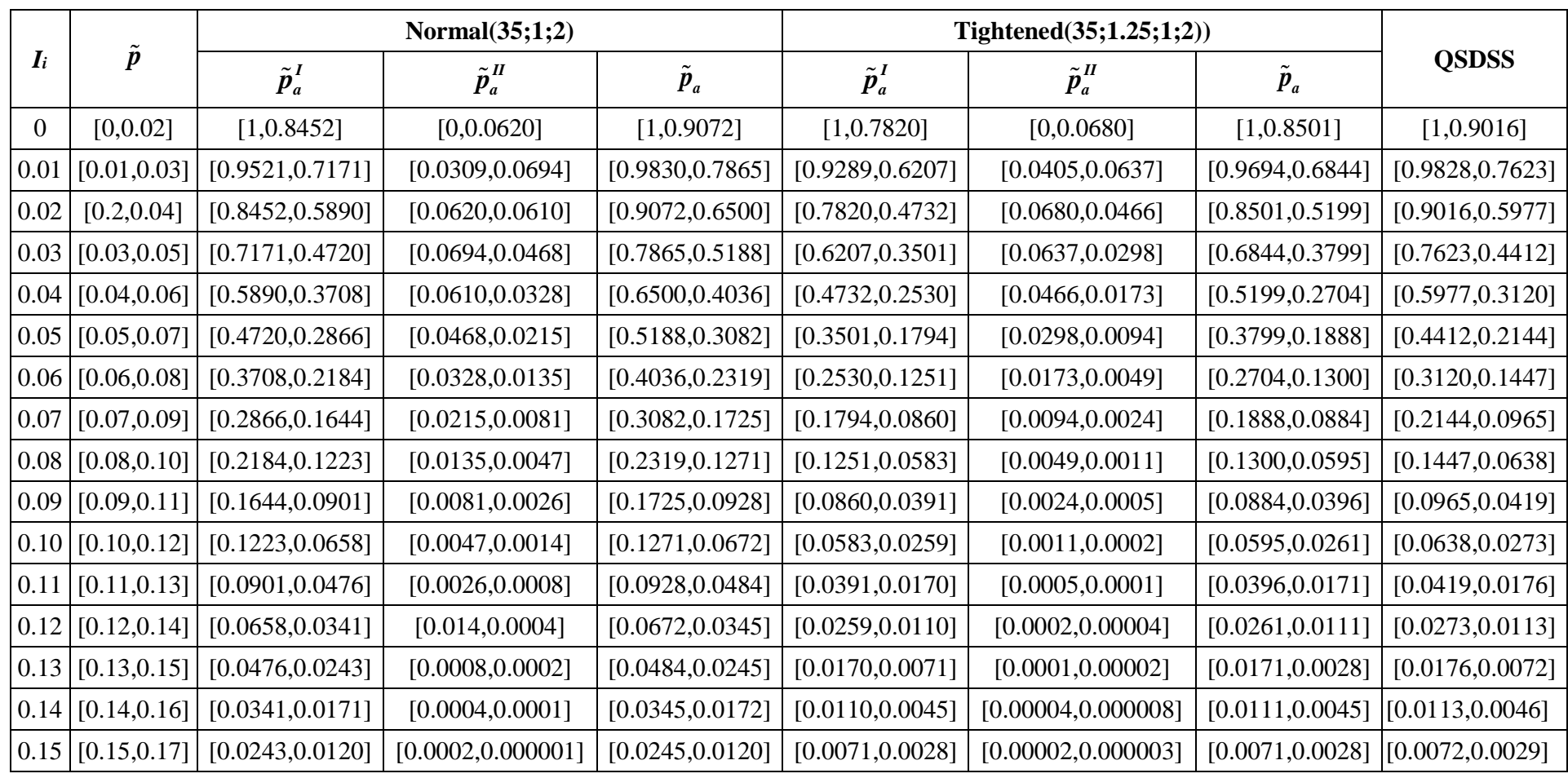

\section{EXAMPLE}

Suppose a product is dispatched with lot size (approximately 1000). If it is fuzzy, the experts in the industry have different suggestions. And according to their suggestions, the average of the sample size is considered as 35 for first and second samples. The average of the acceptance numbers are also considered as (approximately 1 and 2) for the first and second samples respectively. Since the Quick Switching System sample size tightening is used to solve the problem it is taken as 1.25.

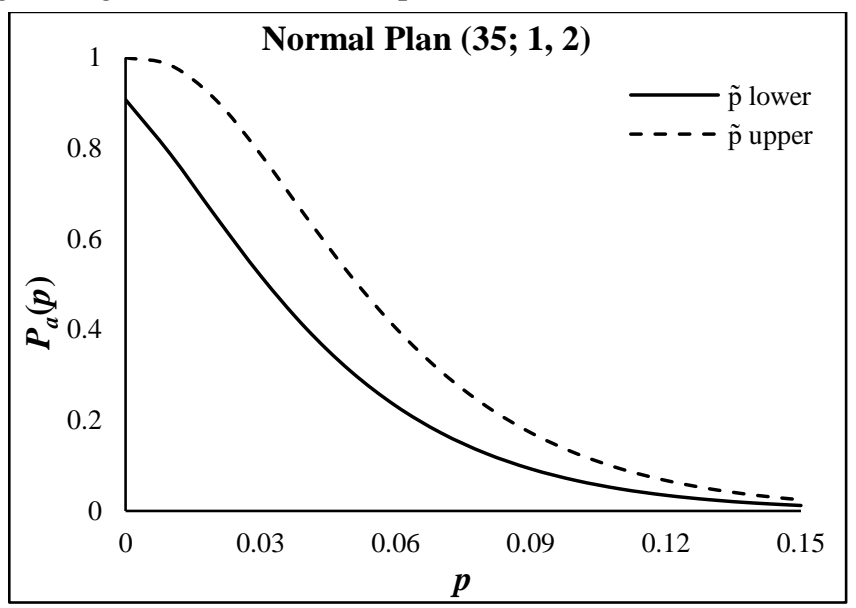

Fig.1. OC curve for Normal Plan

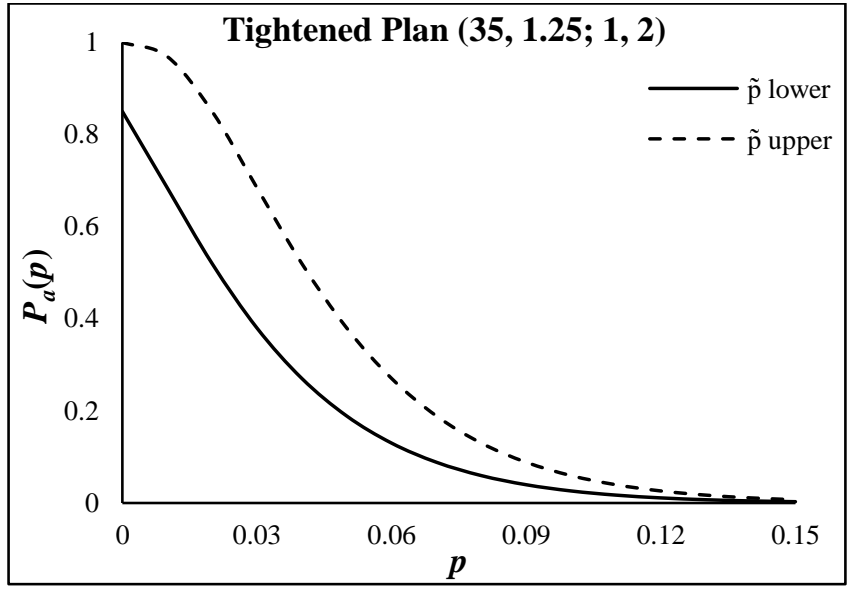

Fig.2. OC curve for Tightened Plan

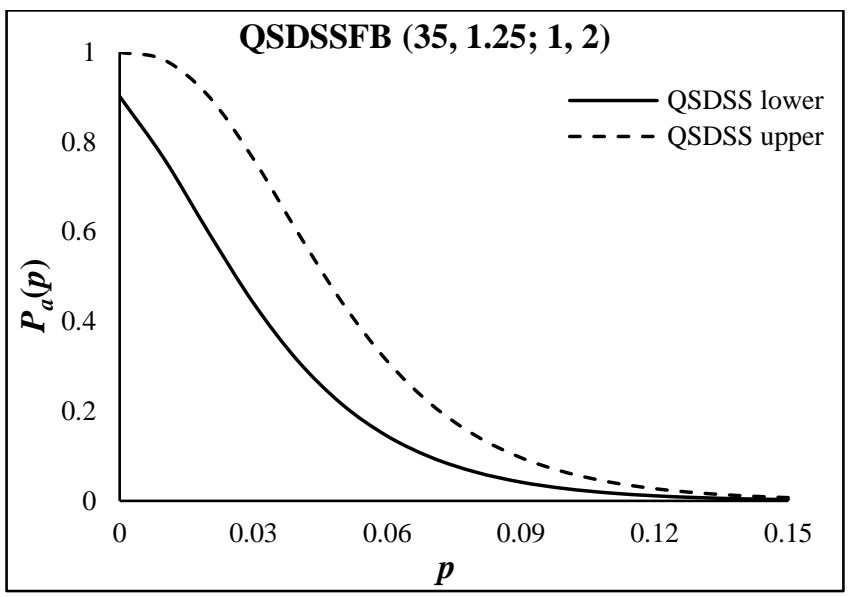

Fig.3. OC curve for $\mathrm{QSDSS}_{\mathrm{FB}}$ 


\section{CONCLUSION}

In this study, fuzzy probability theory is used to solve problems of impreciseness arising in Statistical Quality Control especially in the sampling systems. Also proposed a method for designing Quick Switching Double Sampling System - $\operatorname{QSDSS}_{F B}\left(n, k ; c_{1}, c_{2}\right)$ under sample size tightening using Fuzzy Binomial Distribution. These systems are well defined since if the fraction of defective items is crisp, they reduce to classical plans and system. The uncertainty degree of a proportion parameter is one of the factors that bandwidth depends on that. The less uncertainty value results in less bandwidth, and greater uncertainty values results in wider bandwidth. From this it is suggested that, this system is adopted to predict the uncertainty level in an easy way. Based on this system, the better outcome can be achieved in the shop floor situations.

\section{REFERENCES}

[1] S.D. Arumainayagam and V. Soundararajan, "Quick Switching Double Sampling System (QSDSS)-Acceptance Number Tightening", Communications in Statistics-Simulation and Computation, Vol. 24, No. 3, pp. 2079-2100, 1995.

[2] S.D. Arumainayagam and V. Soundararajan, "Construction and Selection of Quick Switching Double Sampling System Size Tightening", Journal of Applied Statistics, Vol. 22, No. 1, pp. 105-119, 1995.

[3] B.S. Gildeh, G. Yari and E.B. Jamkhaneh, "Acceptance Double Sampling Plan with Fuzzy Parameter", Proceedings of $11^{\text {th }}$ Joint Conference on Information Sciences, pp. 1-9, 2008.

[4] E.B. Jamkhaneh, B.S. Gildeh and G. Yari, "Acceptance Single Sampling Plan with Fuzzy Parameter", Iranian Journal of Fuzzy Systems, Vol. 8, No. 2, pp. 47-55, 2009.

[5] T.K. Chakraborty, "A Class of Single Sampling Plans based on Fuzzy Optimization", Fuzzy Sets and Systems, Vol. 63, No. 1, pp. 35-43, 1994.

[6] T.K. Chakraborty, "Possibilistic Parameter Single Sampling Inspection Plans", Opsearch, Vol. 31, No. 2, pp. 108-126, 1994.

[7] S.D. Arumainayagam and G. Uma, "Construction and Selection of Quick Switching Multiple Sampling SystemSample Size Tightening", Quality Control and Applied Statistics, Vol. 34, No. 2, pp. 115-119, 2011.

[8] P.R. Divya, "Quality Interval Acceptance Single Sampling Plan with Fuzzy Parameter using Poisson Distribution", International Journal of Advancements in Research and Technology, Vol. 1, No. 3, pp. 1-11, 2012.

[9] H.F. Dodge and H.G. Romig, "Sampling Inspection Tables", John Wiley and Sons, 1967

[10] E.B. Jamkhaneh, B.S. Gildeh and G. Yari, "Acceptance Single Sampling Plan with Fuzzy Parameter with the using of Poisson Distribution", World Academy of Science, Engineering and Technology, Vol. 3, No. 1, pp. 1-20, 2009.

[11] E. Turanoglu, I. Kaya and C. Kahraman, "Fuzzy Acceptance Sampling and Characteristic Curves", International Journal of Computational Intelligence Systems, Vol. 5, No. 1, pp. 1329, 2012.

[12] E.B. Jamkhaneh and B.S. Gildeh, "Acceptance Double Sampling Plan using Fuzzy Poisson Distribution", World
Applied Sciences Journal, Vol. 16, No. 11, pp. 1578-1588, 2012.

[13] R. Sampath Kumar, R. Vijaya Kumar and R. Radhakrishnan, "Selection of Mixed Sampling Plans with Conditional Double Sampling Plan as Attribute Plan Indexed through MAPD and LQL using IRPD", International Journal of Computational Engineering Research, Vol. 2, No. 2, pp. 306313, 2012.

[14] O. Hryniewisz, "Statistical Decisions with Imprecise Data and Requirements", Proceedings of International Conference on Systems Analysis and Decisions Support in Economics and Technology, pp. 135-143, 1994.

[15] O. Hryniewisz, "Statistics with Fuzzy Data in Statistical Quality Control", Soft Computing, Vol. 12, No. 3, pp. 229234, 2008.

[16] A. Kanagawa and H. Ohta, "A Design for Single Sampling Attribute Plan based on Fuzzy Sets Theory", Fuzzy Sets and Systems, Vol. 37, No. 2, pp. 173-181, 1990.

[17] A. Kaufmann and M.M. Gupta, "Fuzzy Mathematical Models in Engineering and Management Science", Elsevier, 1988.

[18] V. Palanivel and M. Soundararajan, "Quick Switching Variables Single Sampling (QSVSS) System Indexed by AQL and AOQL", Journal of Applied Statistics, Vol. 27, No. 6, pp. 771-778, 2000.

[19] S. Muthulakshmi and D. Malathi, "Special Double Sampling Plan with Fuzzy Parameter", Indian Journal of Applied Research, Vol. 2, No. 1, pp. 141-143, 2012.

[20] H. Ohta and H. Ichihashi, "Determination of Single Sampling-Attributes Plans based on Membership Functions", International Journal of Production Research, Vol. 26, No. 9, pp. 1477-1485, 1988.

[21] Palanivel, "Results and Tables relating to QSVSSAcceptance Criterion Tightening, Sample Size Tightening", Ph.D Dissertation, Bharathiar University, 1999.

[22] L.D. Romboski, "An Investigation of Quick Switching Acceptance Sampling System", Ph.D Dissertation, RutgersThe State University, 1969.

[23] E.G. Schiling and D.V. Neubauer, "Acceptance Sampling Quality Control", $3^{\text {rd }}$ Edition, CRC Press, 2017.

[24] K.K. Suresh and S. Jayalakshmi, "Selection of Quick Switching System with Special Type Double Sampling Plans through MAPD and MAAOQ", International Journal of Statistics and Management Systems, Vol. 3, No. 12, pp. 93100, 2008.

[25] F. Tamaki, A. Kanagawa and H. Ohta, "A Fuzzy Design of Sampling Inspection Plans by Attributes", Japanese journal of Fuzzy Theory and Systems, Vol. 3, No. 4, pp. 315-327, 1991.

[26] Uma and S.D. Arumainayagam, "Quick Switching Multiple Sampling System-QSMSS", Proceedings of $25^{\text {th }}$ Conference of Indian Society for Probability and Statistics and Annual Meeting of Indian Bayesian Society, pp. 14-18, 2005.

[27] G. Uma and Komaladevi, "Designing Quick Switching System with Fuzzy Parameter", Proceedings of UGC sponsored National Conference on Advances in Statistical Theory, Modeling and Applications, pp. 23-26, 2014.

[28] G. Uma and R. Nandhini Devi, "Determination of Quick Switching System by Attributes under Fuzzy Poisson Distribution", Automation and Autonomous System, Vol. 7, No. 7, pp. 122-126, 2015. 